\title{
Xenobiotic metabolism is triggered in atopic dermatitis
}

Katharina Schwabenbauer ${ }^{1}$, Andreas Elentner ${ }^{1}$, Robert Gruber ${ }^{1}$, Martin Hermann², Barbara Del Frari ${ }^{3}$, Matthias Schmuth ${ }^{1}$, Sandrine Dubrac $^{1}$

1. Department of Dermatology, Venereology and Allergology, Medical University of Innsbruck, Innsbruck, Austria.

2. KMT Laboratory, Department of Visceral, Transplant and Thoracic Surgery, Center for Operative Medicine, Medical University of Innsbruck, Innsbruck, Austria.

3. Department of Plastic, Reconstructive and Esthetic Surgery, Medical University of Innsbruck, Innsbruck, Austria.

Background: Pollution is known to exacerbate asthma and atopic dermatitis (AD). Moreover, mice overexpressing xenobiotic receptors such as Aryl hydrocarbon receptor (AHR) (Hidaka et al., Nat Immunol. 2017) or pregnane x receptor (PXR) (Elentner et al, J Invest. Dermatol. in Press), in the epidermis develop AD-like symptoms. Furthermore, we have found increased expression of genes involved in xenobiotic metabolism in the skin of patients with AD but not with ichthyosis vulgaris (IV) by using RNA sequencing technology (Blunder et al, J Invest. Dermatol. in Press).

Goal of the study: To further investigate the possible link between enhanced metabolism of noxious molecules in AD and to validate data from our RNA sequencing analysis, we performed immunohistochemistry and real-time PCR on skin biopsies from AD patients with wild type or mutated filaggrin or from patients with IV or from healthy controls.
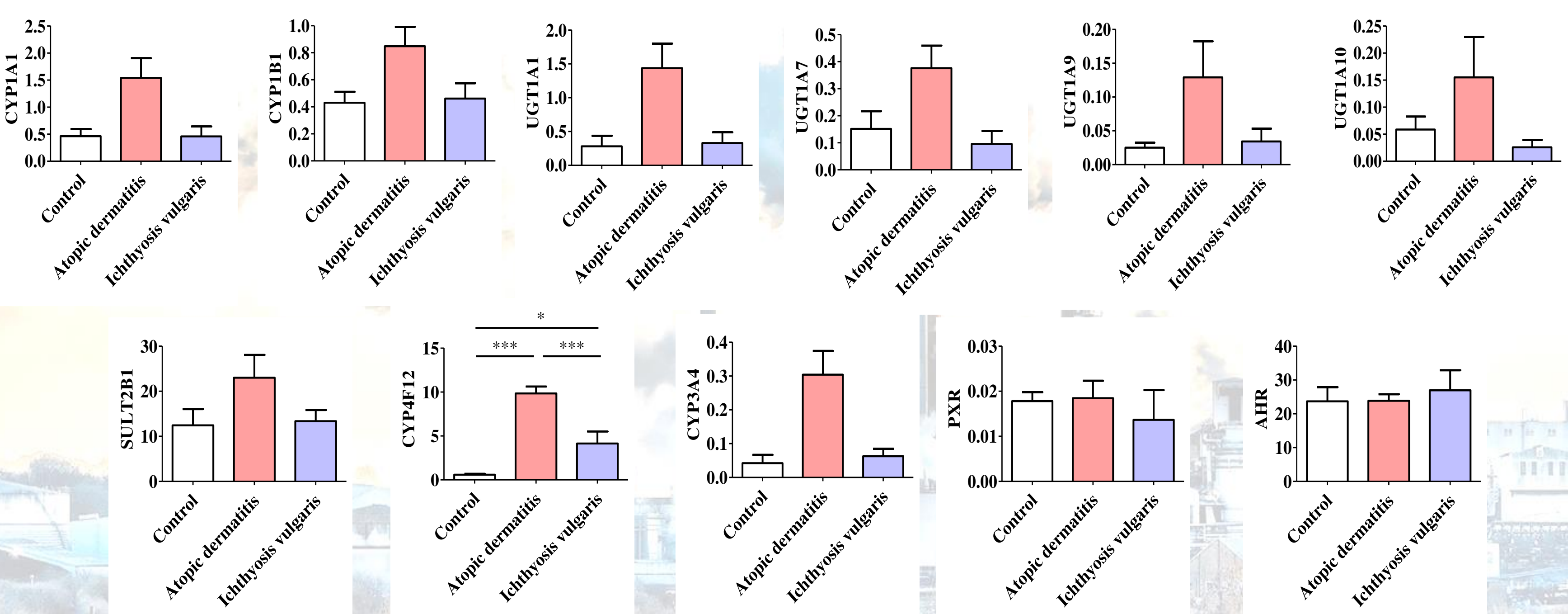

Histograms show changes in expression of various xenobiotic/pollutant - related genes in the skin of patients with AD ( $\mathrm{n}=14$ ) or IV $(n=7)$ when compared to healthy controls $(n=5-9)$. Data were analyzed using a one-way ANOVA test followed by a Tukey post-hoc test with * p-value $<0.05$, *** p-value $<0.0001$. No significant differences between $F L G$ wild type and FLG mutated AD patients could be evidenced. All presented genes are PXR downstream genes.

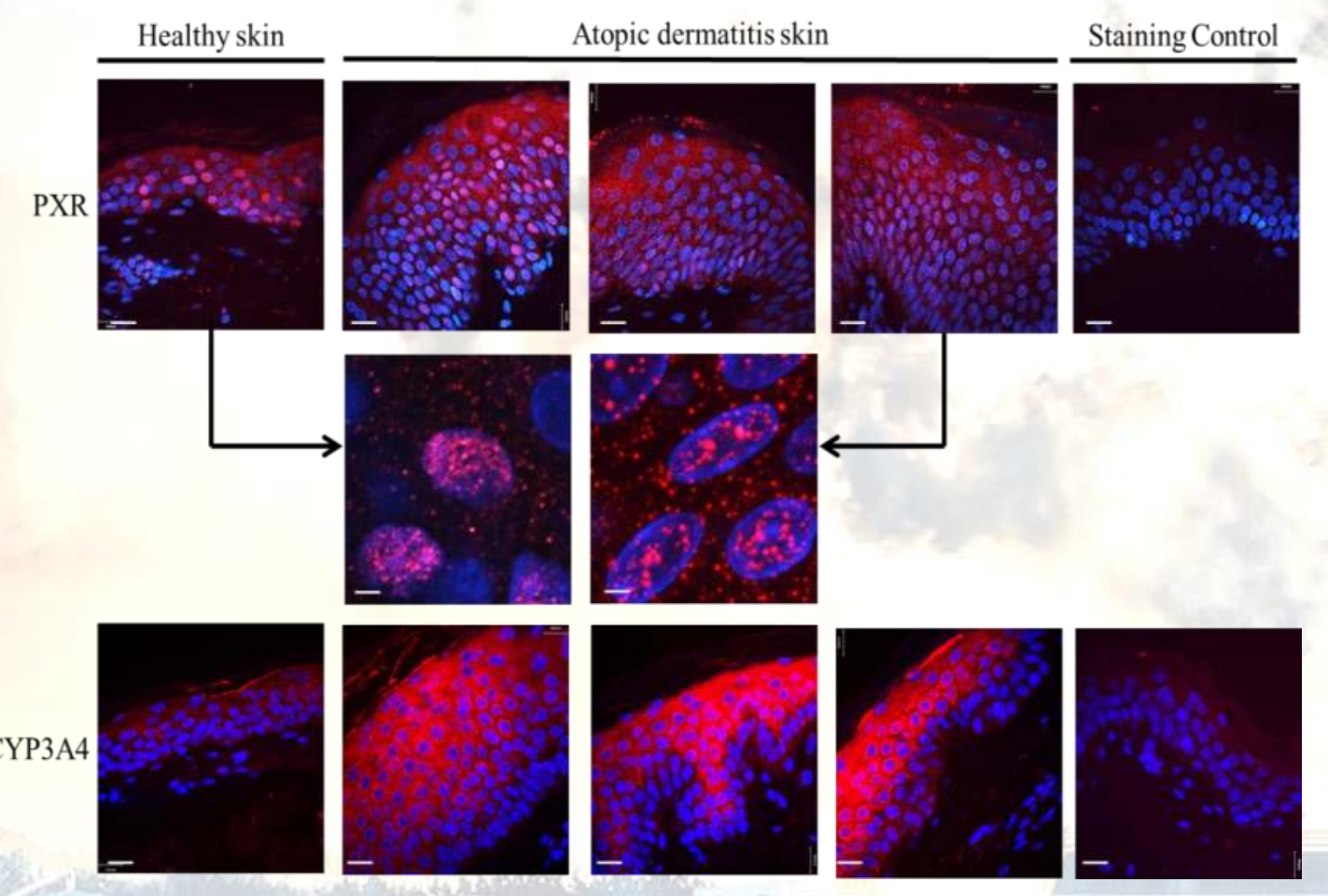

Representative confocal microscopy pictures showing PXR or CYP3A4 expression (red) in skin biopsies from patients with $\mathrm{AD}$ and from healthy donors, $(n=4-8)$. Nuclei are counterstained with DAPI. Rabbit IgG at identical concentrations than primary antibodies has been used to perform staining controls. Bar $=16$ or $1.6 \mathrm{~mm}$.

PXR localizes in both the nucleus and the cytoplasm of basal and suprabasal keratinocytes in healthy human skin. In AD skin, PXR expression changes to a very dotty pattern suggesting an increased transcriptional activity. In line, the expression of CYP3A4, a well-known PXR downstream genes, is markedly increased in suprabasal layers of $\mathrm{AD}$ patients epidermis when compared to healthy controls.

Conclusions: Increased xenobiotic metabolism in the skin, resulting from systemic or topical exposure, might trigger cellular processes such as oxidative stress and cell apoptosis, ultimately leading to or sustaining skin inflammation. Furthermore, data from this work should encourage campaigns to drastically reduce exposure of at-risk populations such as pregnant women and children to xenobiotics. However, the mechanisms by which increased glucuronidation of drugs, pesticides, chemicals and endocrine disruptors trigger skin inflammation remain to be elucidated. 